\title{
Think hard before expanding cancer screening
}

\author{
Fiona Godlee editor in chief, The BMJ
}

How far should we go to find out whether people are infected with hepatitis C? What benefit will this bring them and at what risk? Recommendations for extended population screening are converging on people born between 1945 and 1965. In the United States the Centers for Disease Control and Prevention and the Preventative Services Task Force have recommended this "cohort screening," as most infected people are in that age group. In the US alone this would mean screening 60 million people.

Such intervention might seem easy to justify on the grounds that it would save lives. Hepatitis $\mathrm{C}$ causes chronic illness and premature death from liver failure. And new treatments have emerged that offer hope of clinical benefit. Last year the World Health Organization added its own recommendation to expand screening beyond the usual high risk groups.

But how certain are we that the new treatments are effective and safe? Ronald Koretz and colleagues are not convinced (doi:10.1136/bmj.g7809). Claims for effectiveness are based, they say, mainly on small, short term trials that use surrogate rather than clinical outcomes. And although the new treatments seem to cause fewer adverse events, they are not without serious risk. There is also substantial uncertainty about the natural course of hepatitis $\mathrm{C}$ infection and how this relates to whether or not the virus remains in the blood. Only a small proportion of infected people go on to develop serious complications. When the virus is no longer found in some people's serum, is this the result of treatment, or might it happen anyway? And does this accurately predict whether a person will go on to develop complications? Koretz and colleagues say that we don't yet know.

While these uncertainties persist, the authors say that clinicians should not be pressured to enforce birth cohort screening strategies. What we need, they say, is a large simple trial. Entry to the trial would be at the point of a person's decision on whether or not to be tested for hepatitis C. Those who weren't sure would be invited to be randomised to be tested or not. From that point, treatment would be at the discretion of infected individuals and their clinicians. Data collection would be by linkage to death registries.

This proposed trial design seems a great deal better than an evaluation now under way in the United Kingdom of whether breast cancer screening should be extended to wider age groups. As Ingrid Torjesen explains (doi:10.1136/bmj.h139), as part of a study being run by the UK National Screening Programme some women in their late 40s and over 70 are being invited for screening with the intention of comparing their outcomes with others who are not invited.

Serious concerns about the study were raised in a letter in The $B M J$ last year (doi:10.1136/bmj.g5601), and The BMJ has now learnt of similar concerns raised by the National Research Ethics Committee. Despite recent changes to the protocol, critics still believe that the study is unethical and poor quality: that it is "a randomised controlled clinical trial without a statistical plan or proper consent." They also question whether it is sufficiently independent: its lead investigator, Julietta Patnick, is head of the UK's national screening programme.

While screening for some conditions brings benefit, we should think long and hard before exposing healthy people to potentially harmful medical intervention.

Cite this as: BMJ 2015;350:h226

๑ BMJ Publishing Group Ltd 2015 